\title{
Technè
}

La science au service de l'histoire de l'art et de la préservation des biens culturels

47 | 2019

Bernard Palissy : nouveaux regards sur la céramique française auX $X \mathrm{VI}^{\mathrm{e}}$ et $\mathrm{XVII}{ }^{\mathrm{e}}$ siècles

\section{Corpus des céramiques post-palisséennes de provenance ancienne}

Corpus of post-Palissian ceramics from previous collections

\section{CpenEdition}

Journals

Édition électronique

URL : http://journals.openedition.org/techne/1537

DOI : 10.4000/techne. 1537

ISSN : 2534-5168

Éditeur

C2RMF

\section{Édition imprimée}

Date de publication : 1 juin 2019

Pagination : $72-79$

ISBN : 978-2-11-152830-7

ISSN : 1254-7867

\section{Référence électronique}

"Corpus des céramiques post-palisséennes de provenance ancienne », Technè [En ligne], 47 | 2019, mis en ligne le 01 juin 2020, consulté le 23 juillet 2020. URL : http://journals.openedition.org/techne/ 1537 ; DOI : https://doi.org/10.4000/techne.1537

\section{(c) $(9)$}

La revue Technè. La science au service de l'histoire de l'art et de la préservation des biens culturels est mise à disposition selon les termes de la Licence Creative Commons Attribution - Pas d'Utilisation Commerciale - Pas de Modification 4.0 International. 


\section{Corpus des céramiques post-palisséennes de provenance ancienne}

Corpus of post-Palissian ceramics from previous collections

Ce tableau illustre les vingt-et-une pièces de provenance ancienne prises en compte pour l'étude des céramiques postpalisséennes à la date de cette publication. Au sein du corpus des pièces conservées dans les collections publiques françaises, trois ensembles permettent de remonter aux XVII ${ }^{\mathrm{e}}$ et XVIII ${ }^{\mathrm{e}}$ siècles : douze ouvres provenant des collections de Brunswick et de Cassel, saisies par les armées napoléoniennes, sont actuellement conservées au musée du Louvre, au musée national de la Renaissance à Écouen et au musée d'Art et
d’Archéologie de Guéret ; sur la quinzaine de céramiques dessinées par Étienne de Faye entre 1690 et 1738 dans la Description d'un cabinet et d'un médaillier de l'abbaye Saint-Jeandes-Prémontrés d'Amiens, sept sont encore conservées au musée de Picardie à Amiens ; enfin, deux pièces de la collection constituée par Christophe-Paul de Robien au XviII ${ }^{\mathrm{e}}$ siècle sont arrivées au musée des Beaux-Arts de Rennes par le biais des saisies révolutionnaires.
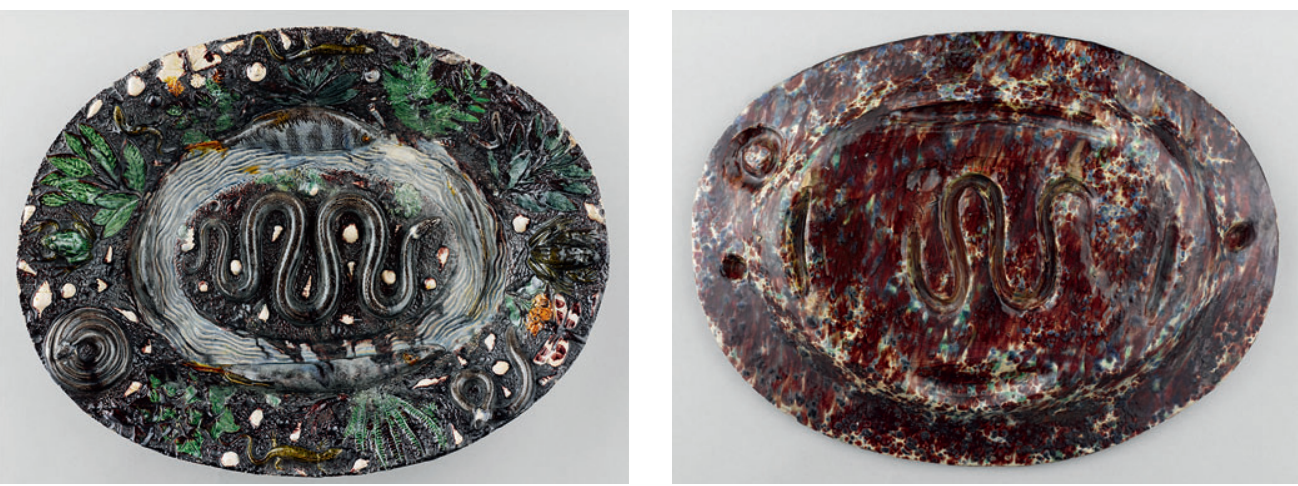

Fig. 1 a et $b$. Plat ovale à décor de rustiques figulines (L. $53 \mathrm{~cm}, 1.40 \mathrm{~cm}, \mathrm{H} .8,8 \mathrm{~cm}$ )

Collection Prince électeur de Hesse à Cassel, Fridericianum Museum ; saisie napoléonienne, 1807 Anciens numéros : $187 \mathrm{~N}^{1} ;$ MR $3529^{2}$

Paris, musée du Louvre, en dépôt au musée national de la Renaissance à Écouen, E.Cl. 13202

๑ RMN-Grand Palais (musée national de la Renaissance, château d'Écouen)/Stéphane Maréchalle.
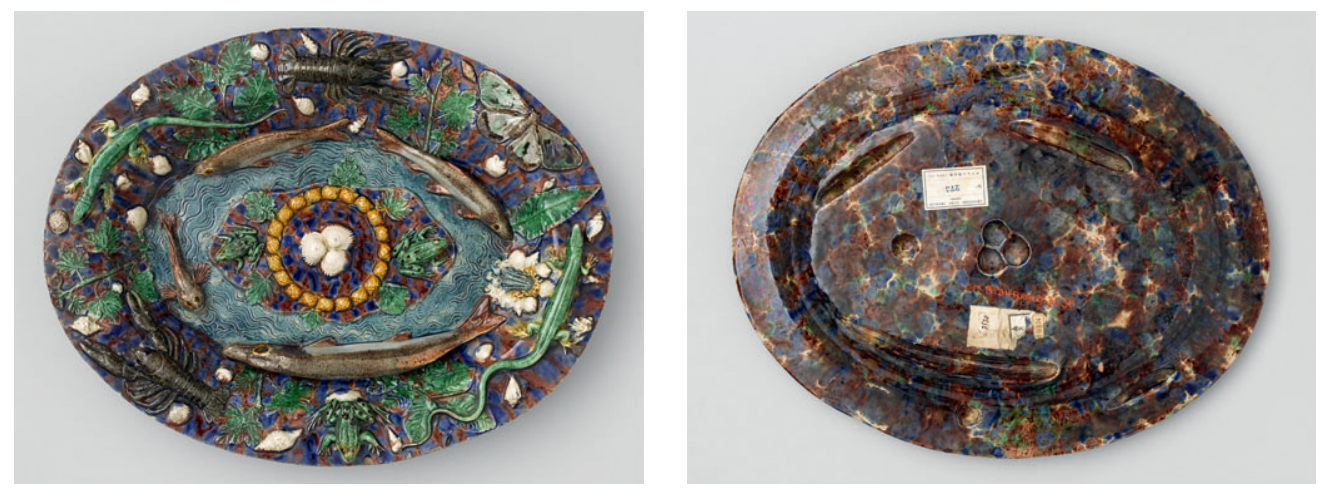

Fig. 2 a et $b$. Plat ovale à décor de rustiques figulines (L. $49,8 \mathrm{~cm}, 1.38,8 \mathrm{~cm}$, H. $6,8 \mathrm{~cm}$ )

Collection Jean-Baptiste Tavernier (1605-1689) ; Anton Ulrich de Brunswick-Wolfenbüttel (1633-1714) ; saisie napoléonienne, 1806

Anciens numéros : Ahrens $342^{3} ; 188 \mathrm{~N}$

Paris, musée du Louvre, MR 3530

(c) RMN-Grand Palais (musée du Louvre)/Jean-Gilles Berizzi. 

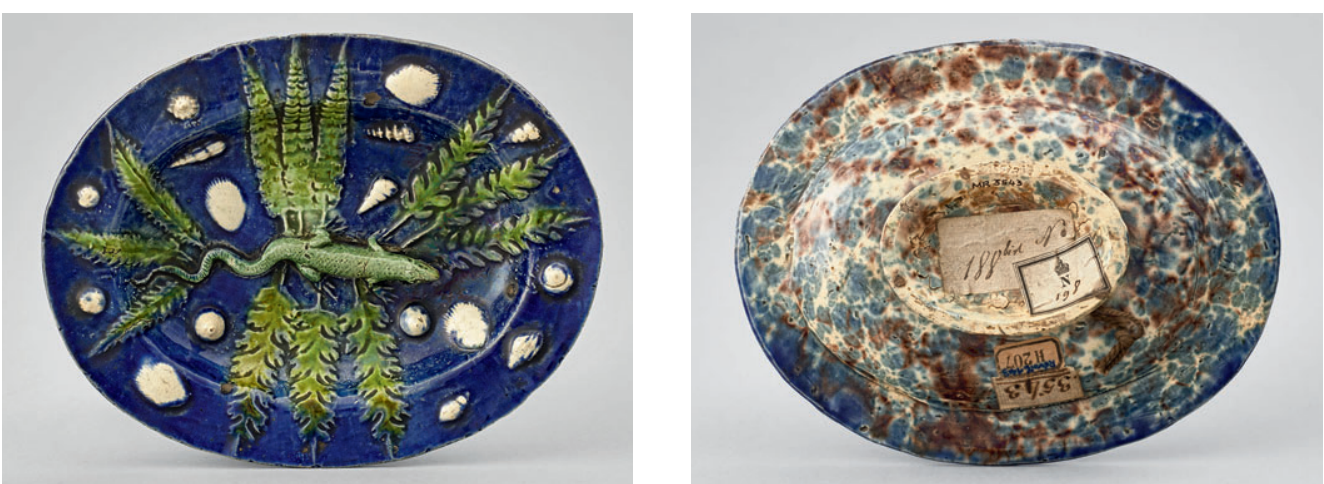

Fig. 3 a et $b$. Plat ovale à décor de rustiques figulines

(L. $17 \mathrm{~cm}, 1.13 \mathrm{~cm}, \mathrm{H} .3 \mathrm{~cm}$ )

Collection Jean-Baptiste Tavernier (1605-1689) ; Anton Ulrich de Brunswick-Wolfenbüttel (1633-1714) ; saisie napoléonienne, 1806

Anciens numéros : Ahrens $350 ; 188 \mathrm{~N}$ bis

Paris, musée du Louvre, MR $3543^{4}$

(C) RMN-Grand Palais (musée du Louvre)/Stéphane Maréchalle.
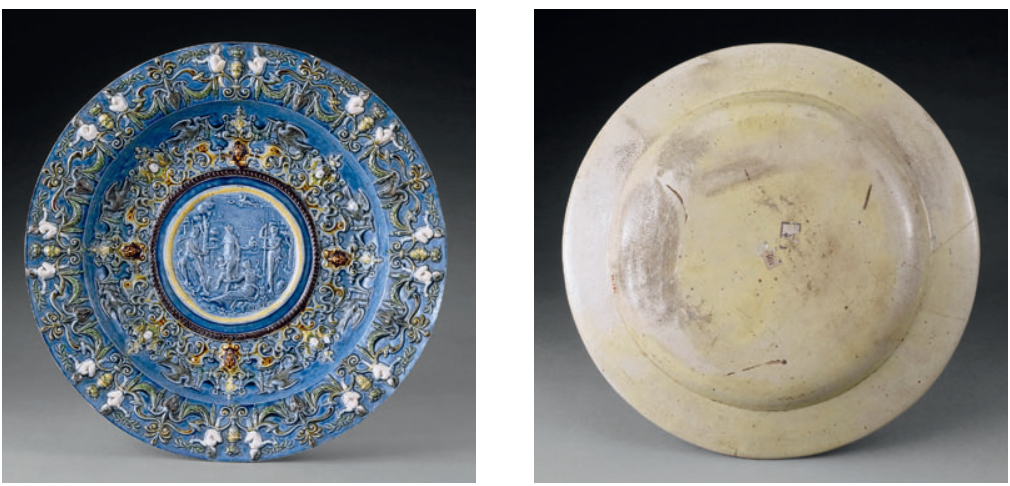

Fig. 4 a et $b$. Plat rond orné de grotesques sur fond bleu avec, au centre, le Jugement de Pâris

(D. $42,5 \mathrm{~cm}$, H. $6,3 \mathrm{~cm}$ )

Collection Jean-Baptiste Tavernier (1605-1689) ; Anton Ulrich de Brunswick-Wolfenbüttel (1633-1714) ; saisie napoléonienne, 1806

Anciens numéros : Ahrens $340 ; 180 \mathrm{~N}$

Paris, musée du Louvre, MR 3535

(C) RMN-Grand Palais (musée du Louvre)/Jean-Gilles Berizzi.
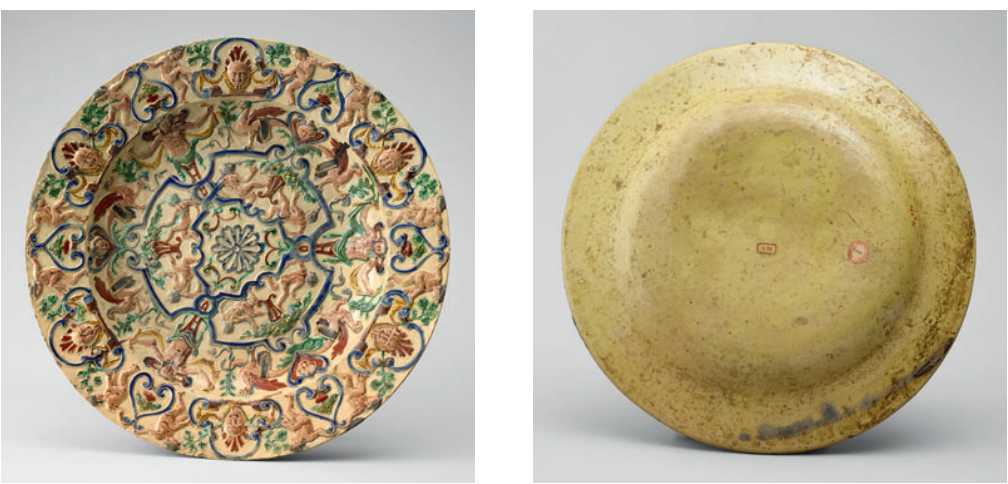

Fig. 5 a et $b$. Plat rond orné de grotesques sur fond beige

(D. $45 \mathrm{~cm}$, H. $6,5 \mathrm{~cm}$ )

Collection Jean-Baptiste Tavernier (1605-1689) ; Anton Ulrich de Brunswick-Wolfenbüttel (1633-1714) ; saisie napoléonienne, 1806

Anciens numéros : Ahrens $647^{5} ; 181 \mathrm{~N}$

Paris, musée du Louvre, MR 3536

(C) RMN-Grand Palais (musée du Louvre)/Stéphane Maréchalle. 

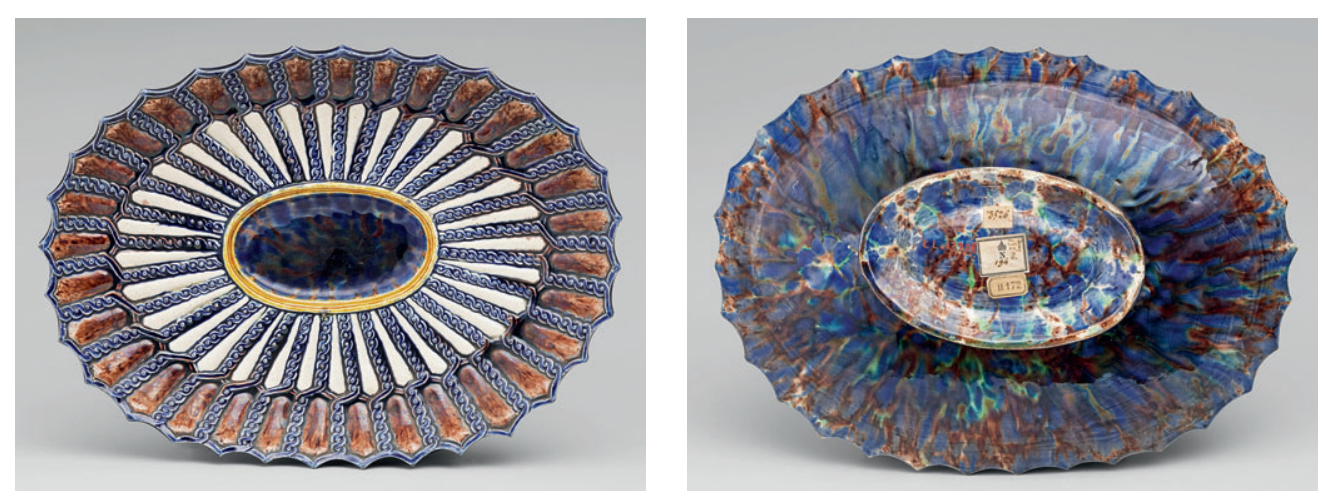

Fig. 6 a et $b$. Coupe ovale ornée d'un décor rayonnant d'entrelacs

(L. $32,3 \mathrm{~cm}, 1.23,7$, H. $5,9 \mathrm{~cm}$ )

Collection Jean-Baptiste Tavernier (1605-1689) ; Anton Ulrich de Brunswick-Wolfenbüttel (1633-1714) ;

saisie napoléonienne, 1806

Anciens numéros : Ahrens $345 ; 186 \mathrm{~N}$; MR 3526

Paris, musée du Louvre, en dépôt au musée national de la Renaissance à Écouen, E.Cl. 13206

๑) RMN-Grand Palais (musée national de la Renaissance, château d'Écouen)/Adrien Didierjean.
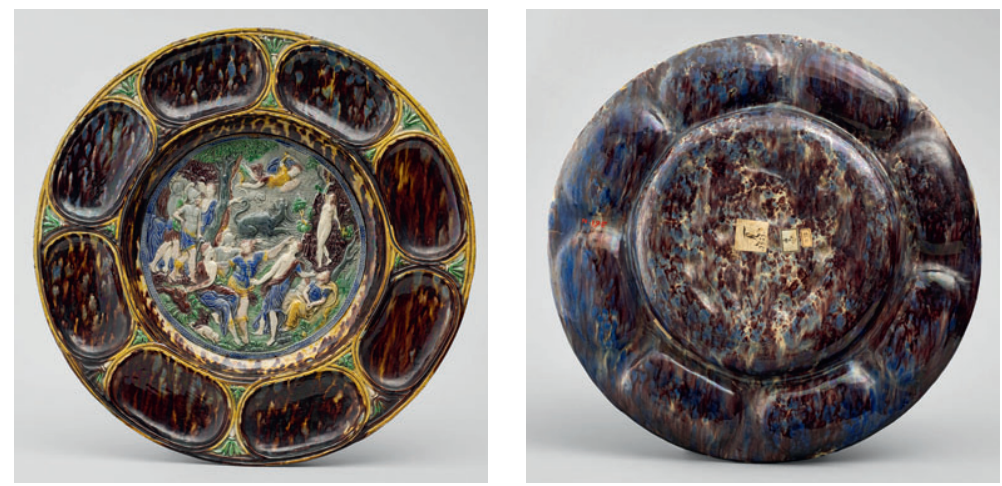

Fig. 7 a et $b$. Plat rond avec Persée et Andromède au centre et huit cavités sur l'aile

(D. $50 \mathrm{~cm}, \mathrm{H} .5,4 \mathrm{~cm}$ )

Collection Jean-Baptiste Tavernier (1605-1689) ; Anton Ulrich de Brunswick-Wolfenbüttel (1633-1714) ; saisie napoléonienne, 1806

Anciens numéros : Ahrens $339 ; 182 \mathrm{~N}$

Paris, musée du Louvre, MR 3525

@ $\mathrm{RMN}-$ Grand Palais (musée du Louvre)/Stéphane Maréchalle.
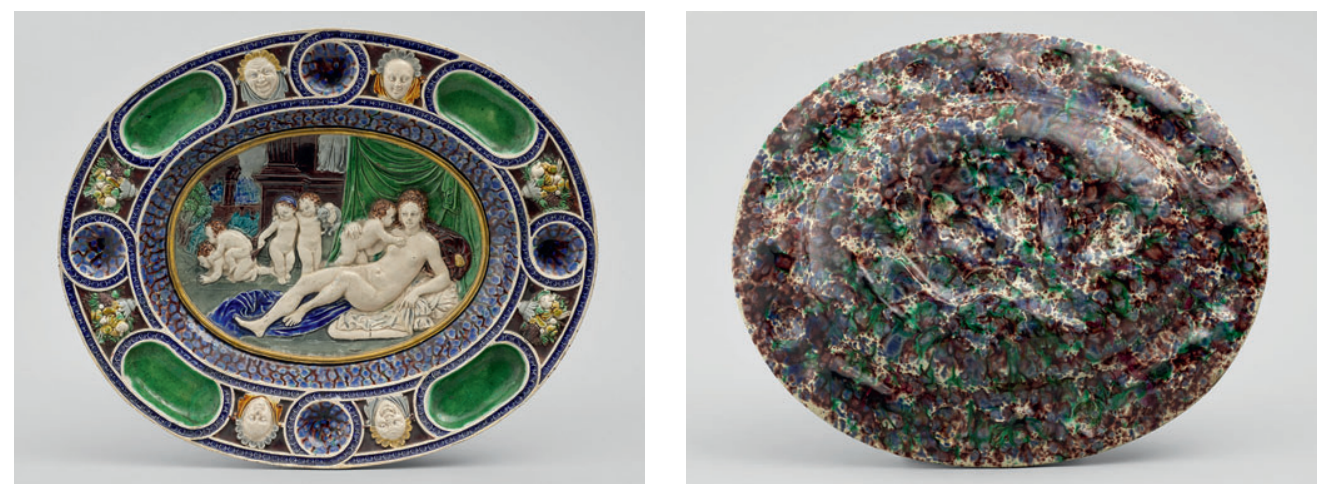

Fig. 8 a et $b$. Plat ovale avec Vénus et les Amours au centre et quatre mascarons et vases alternés sur l'aile (L. $50 \mathrm{~cm}, 1.40,9 \mathrm{~cm}, \mathrm{H} .8,2 \mathrm{~cm}$ )

Collection Prince électeur de Hesse à Cassel, Fridericianum Museum ; saisie napoléonienne, 1807 Ancien numéro : $189 \mathrm{~N}$

Paris, musée du Louvre, MR 3537

๑) RMN-Grand Palais (musée du Louvre)/Stéphane Maréchalle. 

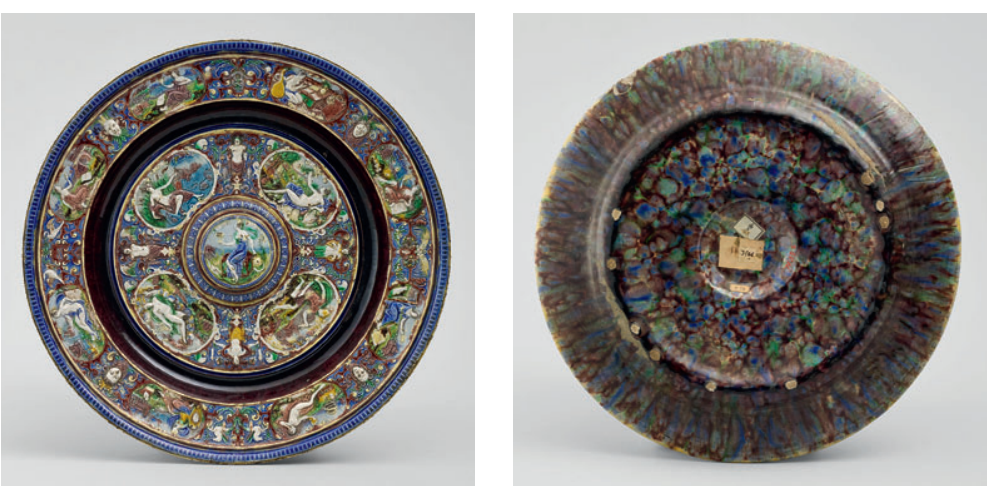

Fig. 9 a et $b$. Bassin d'aiguière, dit « de la Tempérance »

(D. $42 \mathrm{~cm}$, H. $4,2 \mathrm{~cm}$ )

Collection Jean-Baptiste Tavernier (1605-1689) ; Anton Ulrich de Brunswick-Wolfenbüttel (1633-1714) ; saisie napoléonienne, 1806

Anciens numéros : Ahrens $341 ; 183 \mathrm{~N} ; \mathrm{N} 193^{6}$

Paris, musée du Louvre, MR 3528

(C) RMN-Grand Palais (musée du Louvre)/Stéphane Maréchalle.
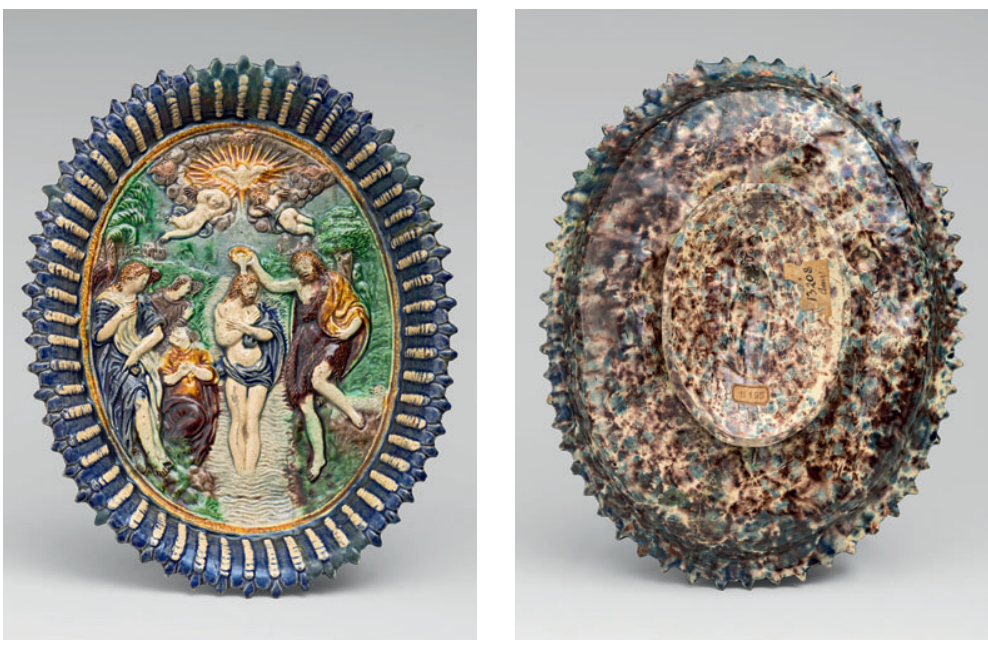

Fig. 10 a et $b$. Plat ovale avec le Baptême du Christ

(L. $29,2 \mathrm{~cm}, 1.23,3 \mathrm{~cm}$, H. $5,6 \mathrm{~cm}$ )

Collection Jean-Baptiste Tavernier(1605-1689) ; Anton Ulrich de Brunswick-Wolfenbüttel (1633-1714) ; saisie napoléonienne, 1806

Anciens numéros : Ahrens 344 ; 189N bis ; MR 3542

Paris, musée du Louvre, en dépôt au musée national de la Renaissance à Écouen, E.Cl. 13208

(c) RMN-Grand Palais (musée national de la Renaissance, château d'Écouen)/Adrien Didierjean.
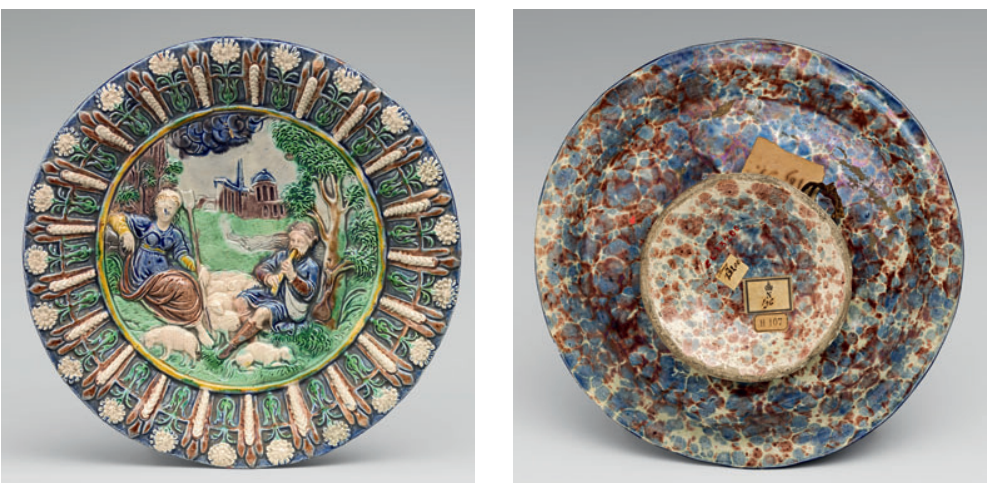

Fig. 11 a et $b$. Coupe avec Bergers et bergères (D. $25,2 \mathrm{~cm}, \mathrm{H} .5 \mathrm{~cm}$ )

Collection Jean-Baptiste Tavernier (1605-1689) ; Anton Ulrich de Brunswick-Wolfenbüttel (1633-1714) ; saisie napoléonienne, 1806

Anciens numéros : Ahrens $348^{7}$; $185 \mathrm{~N} ;$ MR 3532

Paris, musée du Louvre, en dépôt au musée national de la Renaissance à Écouen, E.Cl. 13200

(c) RMN-Grand Palais (musée national de la Renaissance, château d'Écouen)/Adrien Didierjean. 

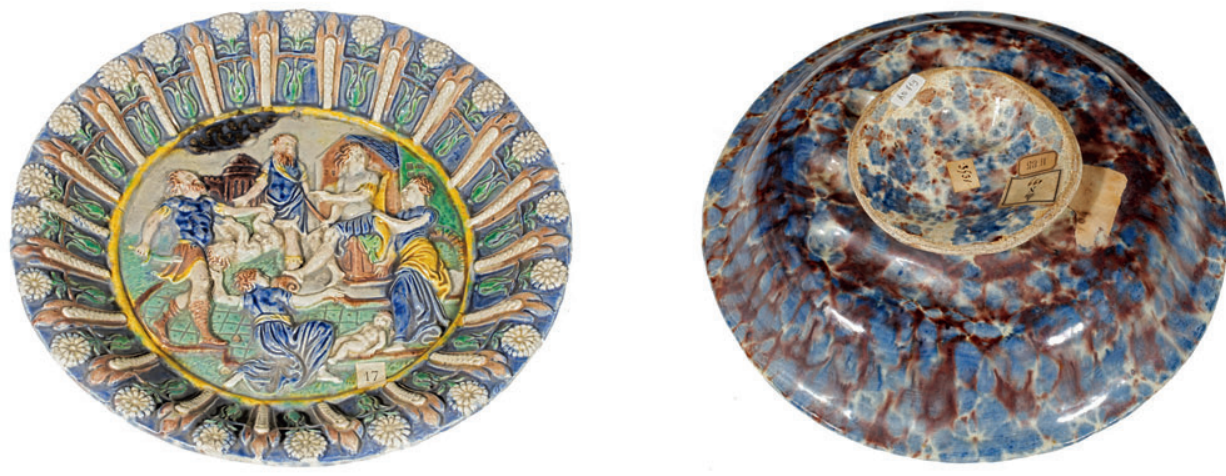

Fig. 12 a et $b$. Plat rond avec le Jugement de Salomon (D. $25,4 \mathrm{~cm}$ )

Collection Jean-Baptiste Tavernier(1605-1689) ; Anton Ulrich de Brunswick-Wolfenbüttel (1633-1714) ;

saisie napoléonienne, 1806 ; dépôt du musée du Louvre au musée de Guéret en 1910 ; transféré en 2009

Anciens numéros : Ahrens 346 ; 184N ; MR 3531

Guéret, musée d'Art et d'Archéologie, 2009.2.58

(c) Musée d'Art et d'Archéologie de Guéret/C. O. Darré.

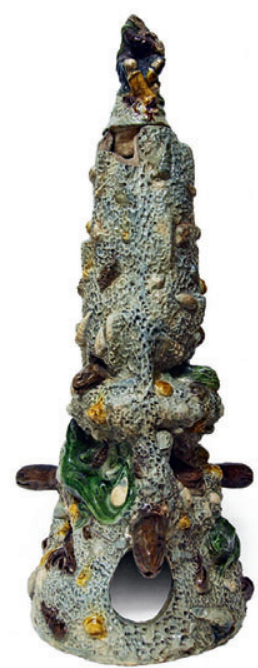

Fig. 13. Fontaine ou rocher rustique

à têtes de murènes, lézards

et coquillages, surmonté d'une

statuette représentant un joueur

de cornemuse

(H. totale $50 \mathrm{~cm}, \mathrm{H}$. sans statuette

$40 \mathrm{~cm}$, L. max. 22,5 cm)

Cabinet de l'abbaye Saint-Jean-des-

Prémontrés d'Amiens

(illustré dans le Ms $400 \mathrm{E}$, vol. 1,

pl. 130, Bibliothèques d'Amiens-

Métropole) ; saisie révolutionnaire,

1790

Amiens, musée de Picardie,

MP 1876.304 bis

(C) C2RMF/A. Maigret.
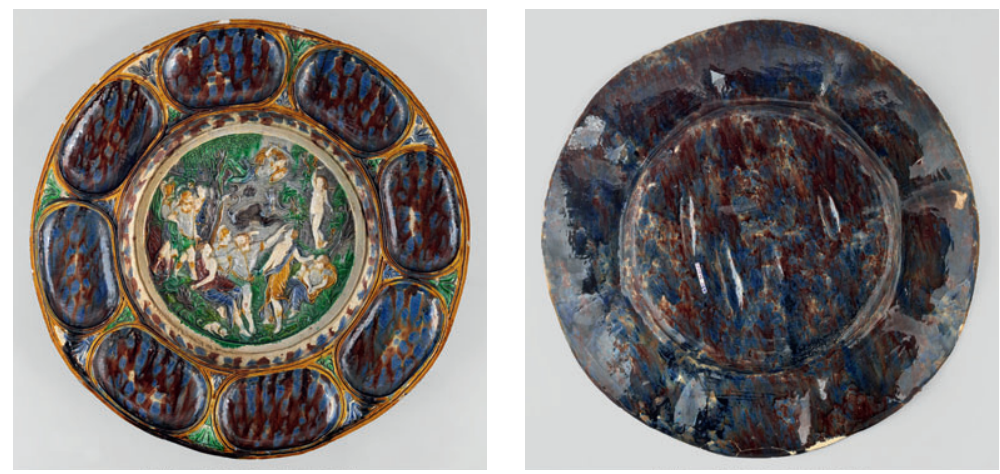

Fig. 14 a et $b$. Grand plat rond avec Persée et Andromède et huit cavités sur l'aile

(D. $50,5 \mathrm{~cm}$, H. $6,2 \mathrm{~cm}$ )

Cabinet de l'abbaye Saint-Jean-des-Prémontrés d'Amiens (illustré dans le Ms 400 E, vol. 1, pl. 132,

Bibliothèques d'Amiens-Métropole) ; saisie révolutionnaire, 1790

Amiens, musée de Picardie, MP 1876.295

(C) C2RMF/A. Maigret. 

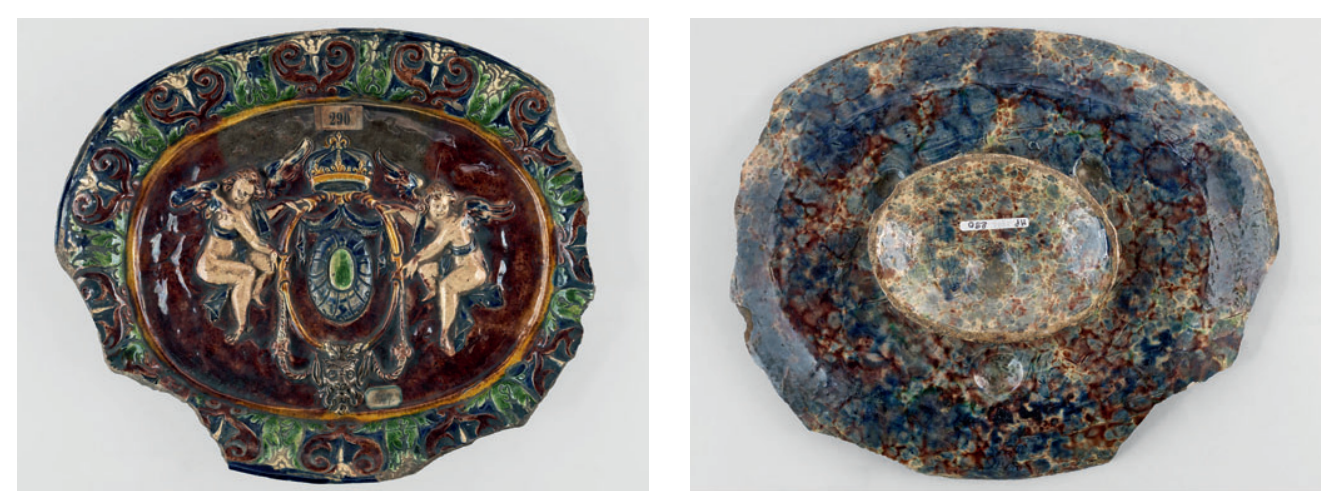

Fig. 15 a et $b$. Plat ovale orné de deux anges et d'une couronne

(L. $28 \mathrm{~cm}, 1.23,8 \mathrm{~cm}$, H. $4,2 \mathrm{~cm}$ )

Cabinet de l'abbaye Saint-Jean-des-Prémontrés d'Amiens (illustré dans le Ms 400 E, vol. 1, pl. 152,

Bibliothèques d'Amiens-Métropole) ; saisie révolutionnaire, 1790

Amiens, musée de Picardie, MP 1876.290

(C) C2RMF/A. Maigret.
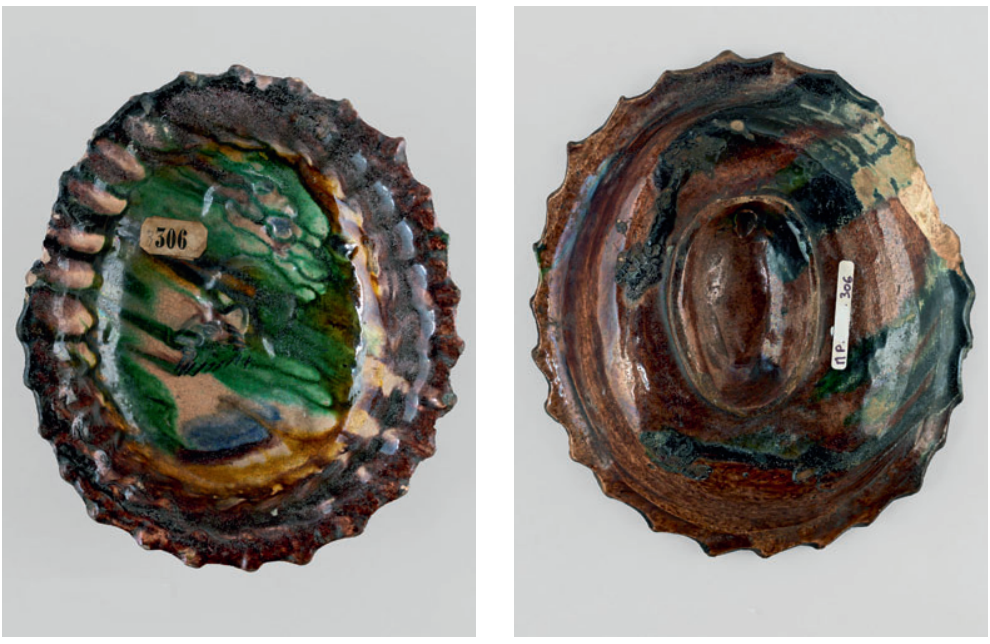

Fig. 16 a et $b$. Plat ovale avec médaillon orné d'un portrait

(L. $17 \mathrm{~cm}, 1.15 \mathrm{~cm}$, H. $5 \mathrm{~cm}$ )

Cabinet de l'abbaye Saint-Jean-des-Prémontrés d'Amiens (illustré dans le Ms 400 E, vol. 1, pl. 152, Bibliothèques d'Amiens-Métropole) ; saisie révolutionnaire, 1790

Amiens, musée de Picardie, MP 306

(c) C2RMF/A. Maigret.
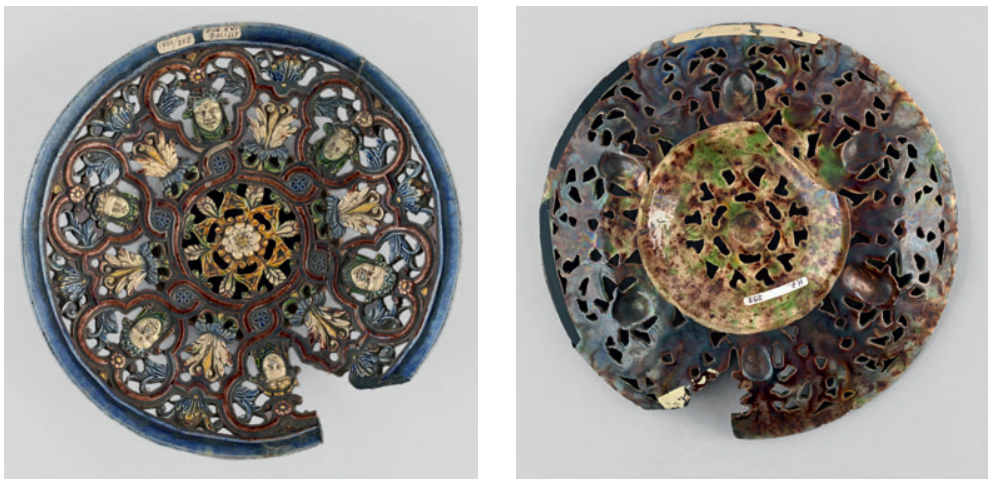

Fig. 17 a et $b$. Plat rond orné d'un décor ajouré avec des têtes de mascarons

(D. $22,8 \mathrm{~cm}$, H. $4,5 \mathrm{~cm}$ )

Cabinet de l'abbaye Saint-Jean-des-Prémontrés d'Amiens (illustré dans le Ms 400 E, vol. 1, pl. 305, Bibliothèques d'Amiens-Métropole) ; saisie révolutionnaire, 1790

Amiens, musée de Picardie, MP 1875.298

(c) C2RMF/A. Maigret. 

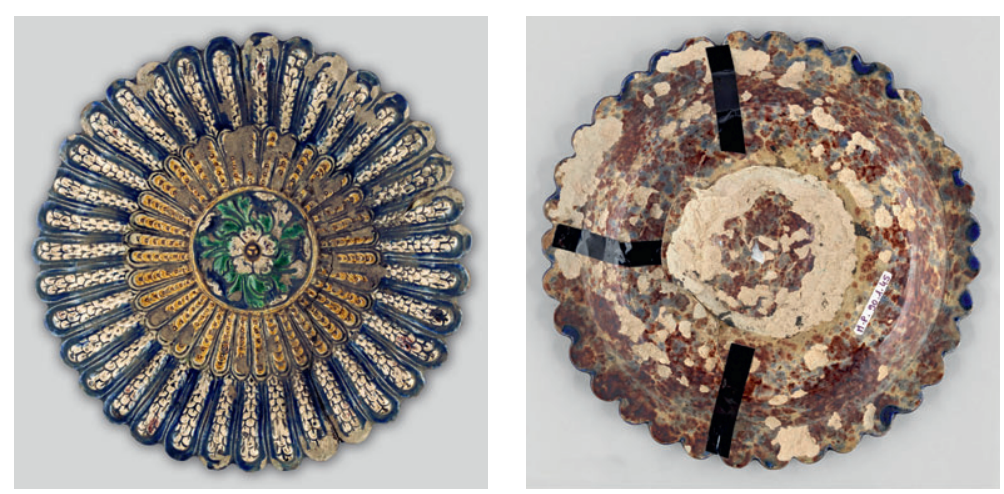

Fig. 18 a et $b$. Plat rond orné d'un décor rayonnant

(D. $23 \mathrm{~cm}, \mathrm{H} .4,5 \mathrm{~cm}$ )

Cabinet de l'abbaye Saint-Jean-des-Prémontrés d'Amiens (illustré dans le Ms 400 E, vol. 1, pl. 302,

Bibliothèques d'Amiens-Métropole) ; saisie révolutionnaire, 1790

Amiens, musée de Picardie, MP 90.1.45

(C) C2RMF/A. Maigret.

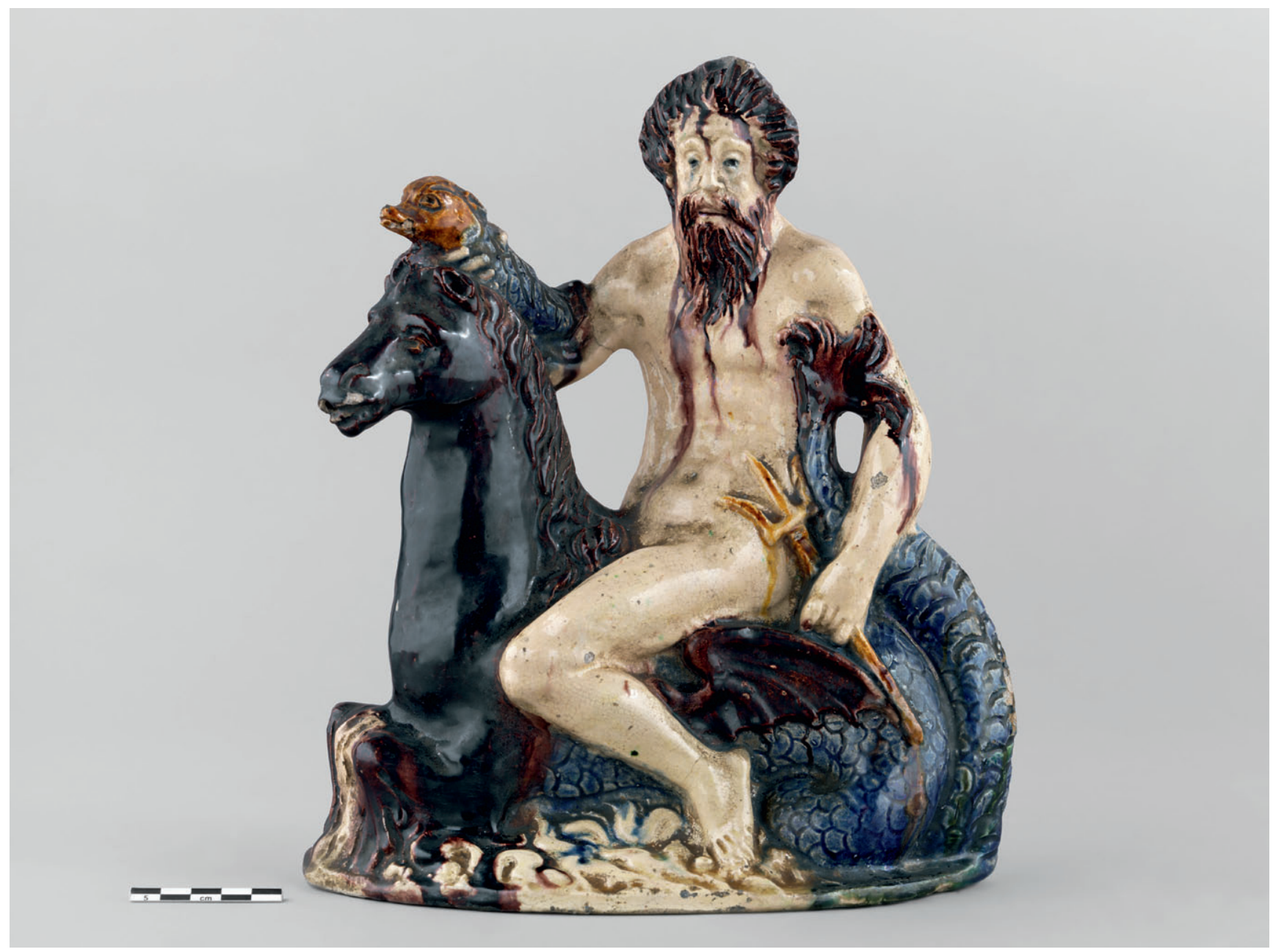

Fig. 19. Statuette: Neptune sur un cheval marin

(H. $28,5 \mathrm{~cm}$, L. $24 \mathrm{~cm}, 1.12,7 \mathrm{~cm}$ )

Cabinet de l'abbaye Saint-Jean-des-Prémontrés d'Amiens (illustrée dans le Ms 400 E, vol. 1, pl. 265 ,

Bibliothèques d'Amiens-Métropole) ; saisie révolutionnaire, 1790

Amiens, musée de Picardie, MP 273

(c) C2RMF/A. Maigret. 

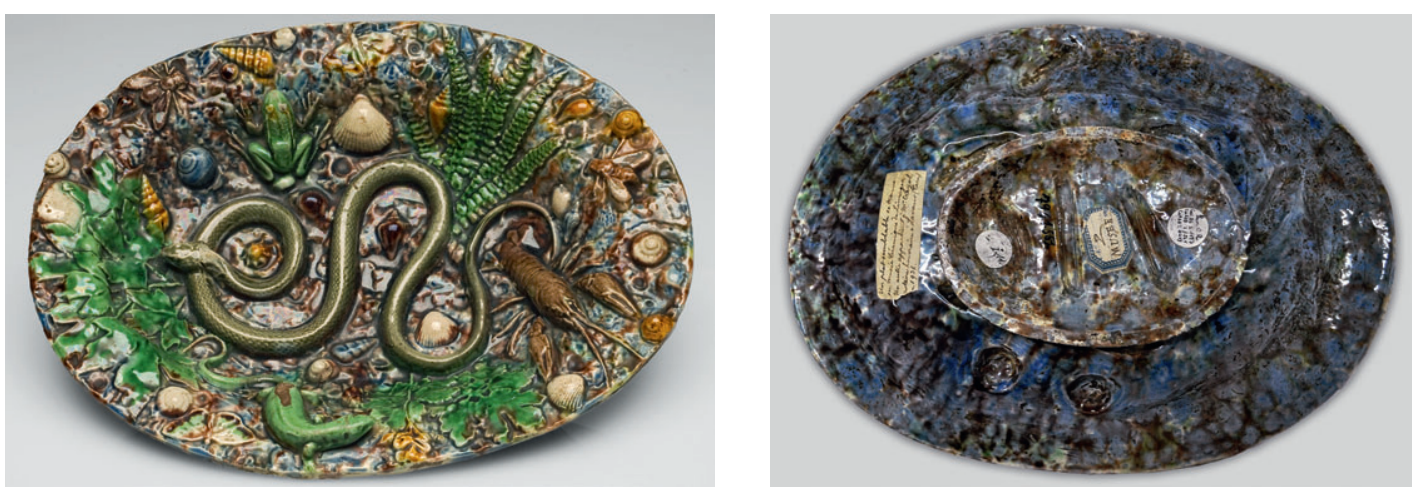

Fig. 20 a et $b$. Plat ovale à décor de rustiques figulines

(L. $31,5 \mathrm{~cm}, 1.24 \mathrm{~cm}$, H. $6 \mathrm{~cm}$ )

Collection Christophe-Paul de Robien (1698-1756) ; saisie révolutionnaire, 1794

Rennes, musée des Beaux-Arts, 794.1.569

(C) Musée des Beaux-Arts de Rennes/J.-M. Salingue (revers : (c) DR.)

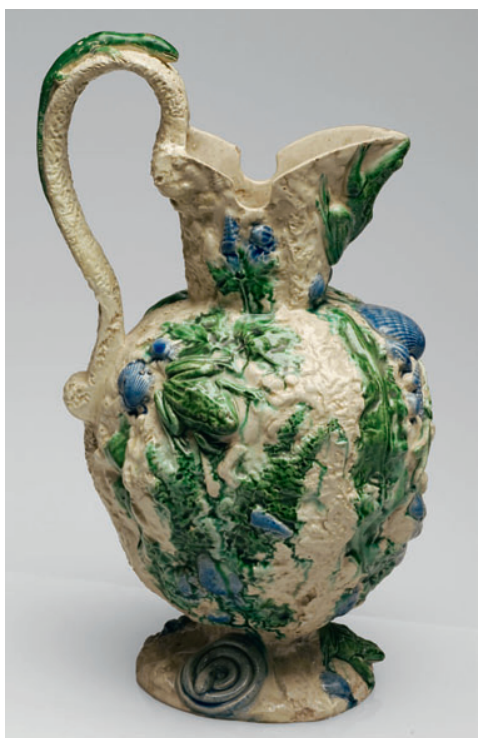

Fig. 21. Aiguière à décor de rustiques figulines

(H. $32 \mathrm{~cm}$, D. $16 \mathrm{~cm}$ )

Collection Christophe-Paul

de Robien (1698-1756) ;

saisie révolutionnaire, 1794

Rennes, musée des Beaux-Arts,

794.1.570

(c) Musée des Beaux-Arts de

Rennes/J.-M. Salingue.

\section{Notes}

1. Les numéros $180 \mathrm{~N}$ à $191 \mathrm{~N}$ proviennent de l'Inventaire du musée Napoléon, 1810, Dessins, t. IX, fol. 1820 à 1823 (A.N., 1 DD 41/2 MI 119).

2. Les numéros MR 3525 à MR 3543 proviennent de l'Inventaire général des musées royaux, 1824, Sculptures, t.5, fol. 221-222 (A.N., 1 DD 80/2 MI 011).

Ce plat a longtemps été considéré, par erreur, comme provenant de la collection Durand, acquise en 1825, sous le n MR 2329 (Inventaire général des musées royaux, 1832, Objets divers, t. 16, A.N., 1 DD 104/2 MI 041) et déposé au musée de Cluny en 1895 .
3. Les numéros 339 à 350 proviennent de l'inventaire H 29 rédigé par Anton Konrad Friedrich Ahrens : Braunschweig, Herzog Anton Ulrich-Museum, Altregistratur, Beschreibung oder Inventarium des Herzogl. Braunschw. Museums. Ersten Band enthaltend, 9, Andere irdene Geschirre.

4. Ce plat a longtemps été considéré, par erreur, comme provenant de la collection Révoil, acquise en 1828, sous le n MRR 143 (Inventaire général des musées royaux, 1832, Objets divers, t. 16, A.N., 1 DD 104/2 MI 041).

5. Contrairement aux autres, ce plat apparaît dans l'inventaire H 33 (faiences italiennes) rédigé par Anton Konrad Friedrich Ahrens : Beschreibung oder Inventarium des Herzoglich Braunschweiischen Museums. Viertel Band enthalten 1. Geschirre von alter italienischer Fayence, Majolica genannt.
6. Le numéro N 193 provient de l'Inventaire du Musée des Souverains Français, 1852-1857, Section Première, Chapitre Deuxième, fol. 20v (A.N., 9 DD 2)

7. Dans l'Inventaire des objets d'art et de curiosité enlevés du musée de Brunswick par le Directeur Général du musée Napoléon, signé le 28 décembre 1806, la coupe apparaît sous le $\mathrm{n}^{\circ} 347$ (archives du musée de Brunswick, H 80 Nr. 2). Il semble qu'il y ait eu une erreur au moment de l'envoi à Paris car le musée de Brunswick possédait trois pièces avec Bergers et bergères (347, 348 et 349), mais seules les dimensions de l'objet 348 correspondent à la pièce conservée à Paris. 